\title{
СТРУКТУРА, ДИЭЛЕКТРИЧЕСКИЕ И ТЕПЛОВЫЕ СВОЙСТВА ТЕТРАХЛОРЦИНКАТА РУБИДИЯ В ПОРИСТЫХ СТЕКЛАХ
}

\author{
(C2019 Л.Н. Коротков ${ }^{1}$, Л.С. Стекленева ${ }^{1}$, И.Н. Флеров ${ }^{2,3}$, Е.А. Михалева ${ }^{2}$, Е. Rysiakiewicz- \\ Pasek $^{4}$, М.С. Молокеев ${ }^{2,3}$, В.С. Бондарев ${ }^{2,3}$, М.В. Горев ${ }^{2,3}$, О.И. Сысоев ${ }^{5}$
}

${ }^{1}$ Федеральное государственное бюджетное образовательное учреждение высшего образования «Воронежский государственный технический университет». Воронеж, 394026, Россия.

${ }^{2}$ Институт физики им. Л.В. Киренского Сибирского отделения Российской академии наук обособленное подразделение ФИЦ КНЦ СО РАН, Красноярск, 660036, Россия

${ }^{3}$ Институт Инженерной Физики и Радиоэлектроники, Сибирский федеральный Университет, Красноярск, 660074, Россия

${ }^{4}$ Division of Experimental Physics, Faculty of Fundamental Problems of Technology, Wroclaw University of Science and Technology, 50-370 Wroclaw, Poland

${ }^{5}$ ВУНЦ ВВС «Военно-воздушная академия имени профессора Н.Е. Жуковского и Ю. А. Гагарина», ул. Старых Большевиков, 54 «А», г. Воронеж, 394064, Россия

e-mail: lubov_stekleneva@mail.ru

Представлены результаты исследования рентгеновской дифракции, теплоемкости $C_{\mathrm{p}}(T)$, диэлектрической проницаемости и коэффициента объемного теплового расширения композиционных материалов, полученных внедрением соли $\mathrm{Rb}_{2} \mathrm{ZnCl}_{4}$ в матрицы из боросиликатного стекла со средним диаметром пор 46 и 320 нм для интервала температур 120-350 К. Анализ полученных данных позволил определить температуры переходов в несоразмерную и сегнетоэлектрическую фазы, а также температуру замораживания подвижности доменных границ в частицах $\mathrm{Rb}_{2} \mathrm{ZnCl}_{4}$. Обнаружено значительное повышение температуры Кюри $\Delta \mathrm{T}_{\mathrm{C}} \approx 50 \mathrm{~K}$.

В настоящее время в физике твердого тела и физическом материаловедении наблюдается интерес к изучению явлений, связанных с проявлением размерного эффекта в различных материалах, в частности, в системах ультрадисперсных частиц различной топологии и размерности. 
Влияние «ограниченной геометрии» на электрофизические свойства [1-5], структуру $[6,7]$ и фазовые переходы [1-7] в частицах ряда модельных сегнетоэлектриков (TGS, $\mathrm{KH}_{2} \mathrm{PO}_{4}, \mathrm{NH}_{4} \mathrm{HSO}_{4}$ и др.) изучено уже достаточно хорошо. Вместе с тем, физические свойства ультрадисперсных сегнетоэлектриков с несоразмерными фазами, до сих пор практически не были исследованы, за исключением нитрита натрия. Однако выявить существование несоразмерной фазы во внедренных частицах $\mathrm{NaNO}_{2}$ до сих пор не удалось, что, вероятно, связано со слишком узкой температурной областью ее существования (около $1 \mathrm{~K})$.

Существуют подобные материалы с несоразмерной фазой, занимающей более широкую температурную область. Примером является сегнетоэлектрик - сегнетоэластик тетрахлорцинкат рубидия $\left(\mathrm{Rb}_{2} \mathrm{ZnCl}_{4}\right)$. В объемном кристалле переход из параэлектрической в несоразмерную фазу реализуется при температуре $\mathrm{T}_{\mathrm{i}}=303 \mathrm{~K}$, а из несоразмерной в сегнетоэлектрическую - при температуре $\mathrm{T}_{\mathrm{C}}=195 \mathrm{~K}$ [8]. Таким образом, область существования несоразмерной фазы в данном материале составляет около 108 К, что делает его более подходящим объектом для изучения влияния «ограниченной геометрии» на фазовые переходы при $\mathrm{T}_{\mathrm{i}}$ и $\mathrm{T}_{\mathrm{C}}$.

Целью настоящей работы является комплексное изучение влияния «ограниченной геометрии» на физические свойства и кооперативные процессы в тетрахлорцинкате рубидия.

Эксперименты выполнены на поликристаллическом образце тетрахлорцинката рубидия и композитах, полученных путем внедрения соли $\mathrm{Rb}_{2} \mathrm{ZnCl}_{4}$ в стеклянные матрицы в виде пластин с внешними размерами 10х10x0,5 мм и средним диаметром сквозных пор 46 и 320 нм. (В дальнейшем для обозначения этих композитов примем аббревиатуры: RS - 46 и RS-320, соответственно.)

Процесс внедрения материала в предварительно отожженные матрицы осуществлялся из насыщенного водного раствора тетрахлорцинката рубидия при температуре около $100{ }^{\circ} \mathrm{C}$ в течении 3,5 часов. Затем образцы вынимались из раствора и тщательно высушивались при температуре $330{ }^{\circ} \mathrm{C}$ в течение 10 часов. Плотность незаполненных матриц обоих типов составила приблизительно 1,2 г/см³ , относительный объем пор - около 55 \%, объемная доля $\mathrm{Rb}_{2} \mathrm{ZnCl}_{4}$ в порах $\approx 10 \%$, а его доля по отношению к объему всего образца $\approx 6 \%$.

Рентгенодифракционный анализ, проведенный с использованием рентгеновского дифрактометра «D2 PHASER» (длина волны характеристического излучения $\lambda_{\mathrm{Cu}}=1,5418 \AA$ ), показал, что внедренный материал кристаллизовался в порах матрицы в виде кристаллитов со структурой, соответствующей объемному $\mathrm{Rb}_{2} \mathrm{ZnCl}_{4}$. Оценки размеров внедренных 
частиц в порах, сделанные с использованием программы TOPAS 4.2 [9] дали $\mathrm{d} \approx 70$ и 51 нм для RS - 46 и RS-320 соответственно.

Дифракционные спектры для образца RS - 46 были подробно изучены в интервале температур 143 - 320 K, с помощью дифрактометра Bruker D8 ADVANCE (Cu-Kaизлучение), снабженного камерой Anton Paar и линейным детектором VANTEC. Измерения проводили в диапазоне углов $2 \theta=10-90^{\circ}$ с шагом $2 \theta=0,016^{\circ}$, при этом время счета составляло 1 с на шаг.

Для проведения диэлектрических исследований на поверхности образцов были нанесены серебряные электроды. Образцы помещали в криостат, где температура изменялась от 100 до 350 К и контролировалась с помощью платинового термометра сопротивления с погрешностью не превышающей $\pm 0,2$ К. Измерения диэлектрической проницаемости осуществлялось с помощью измерителя иммитанса Е7-12 на частоте 10 кГц в процессах охлаждения и нагрева образца со скоростью около $1 \div 2$ К/мин. Перед каждым измерением для удаления адсорбированной из воздуха воды осуществляли отжиг образцов при температуре $\approx 400 \mathrm{~K}$.

Измерения теплоёмкости $C p(T)$ в широком интервале температур было выполнено на автоматизированном адиабатическом калориметре, описанном в работе [10]. Измерения проводили в вакууме $10^{-6}$ мм рт. ст. в режиме дискретных и непрерывных нагревов. Погрешность определения теплоемкости не превышала $\pm 1.0 \%$.

Тепловое расширение исследовалось на дилатометре DIL-402C в интервале 100-370 K в динамическом режиме со скоростью изменения температуры $3 \mathrm{~K} /$ мин в атмосфере гелия.

Измерения линейного коэффициента теплового расширения стеклянных матриц показали отсутствие аномального поведения, при этом его величина не превышает $\alpha \leq 5 \cdot 10^{-6}$ $\mathrm{K}^{-1}$.

Анализ спектров рентгеновского рассеяния для композита $\mathrm{RS}$ - 46, полученных для интервала температур 140 - 320 К показал (рис. 1a), что ниже $310 \mathrm{~K}$ появляется сверхструктурный пик (2/3 2 0), , соответствующей температуре $\mathrm{T}_{\mathrm{i}}$ объемного $\mathrm{Rb}_{2} \mathrm{ZnCl}_{4}$. Интенсивность пика увеличивается по мере охлаждения образца (рис. 1б). Других сверхструктурных линий в ходе эксперимента не наблюдалось, в отличие от данных работы [8], где изучались монокристаллические образцы тетрахлорцинката рубидия. Это обстоятельство может быть связано с весьма небольшим количеством сегнетоэлектрического компонента в композите.

Определена структура кристаллитов $\mathrm{Rb}_{2} \mathrm{ZnCl}_{4}$ в условиях «ограниченной геометрии» при температурах 320 K, 205 K и 143 K (табл. 1). Сравнение параметров элементарной ячейки при комнатной температуре с данными для монокристаллического образца [8] 
показало их хорошее совпадение. Объем ячейки V кристаллитов, приходящийся на формульное число Z, незначительно уменьшается при охлаждении (табл.), и при всех температурах практически почти совпадает с данными [8].

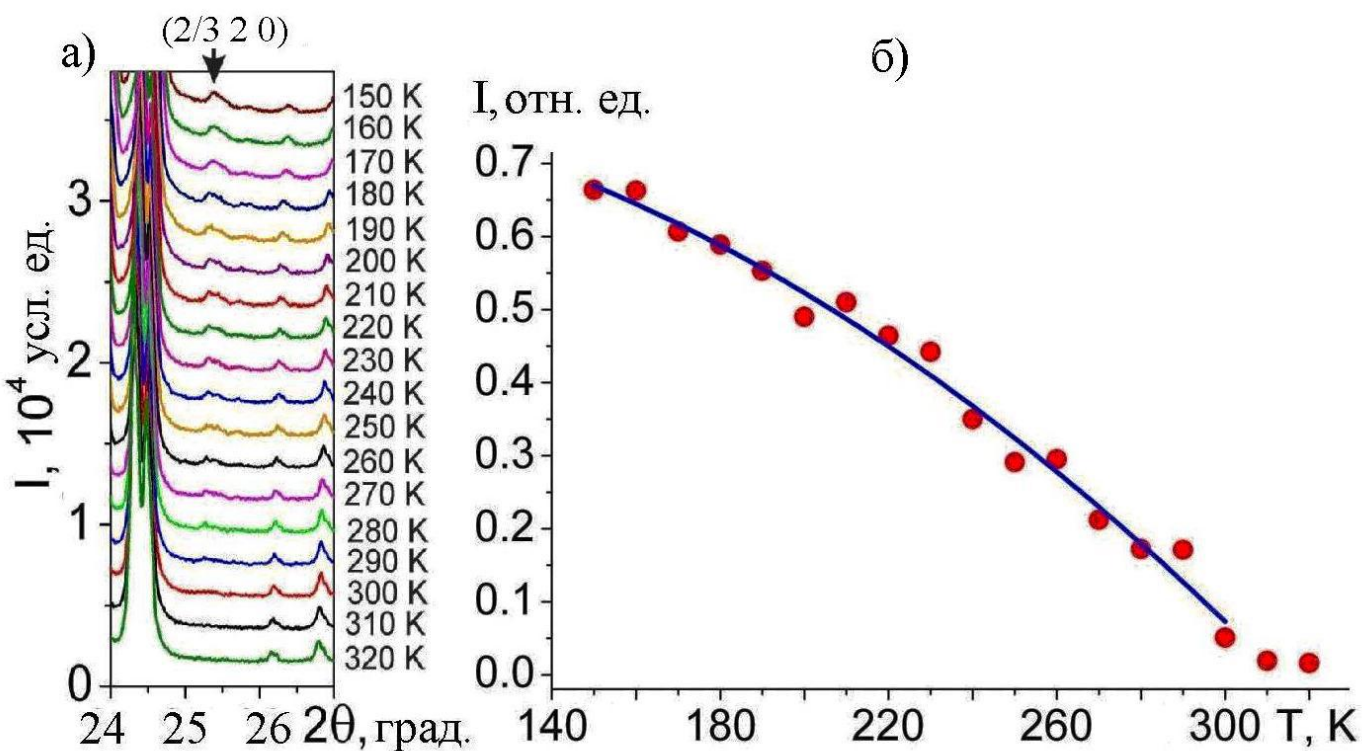

Рис1. а) - Фрагмент рентгеновской дифрактограммы для композита RS - 46 при различных температурах. Стрелкой помечен сверхструктурный пик (2/3 20$)$. б) -Температурная зависимость интенсивности сверхструктурного пика (2/3 2 0).

Таблица 1. Параметры кристаллической структуры $\mathrm{Rb}_{2} \mathrm{ZnCl}_{4}$ в матрице пористого стекла со средним диаметром пор 46 нм

\begin{tabular}{|c|c|c|c|c|}
\hline \multicolumn{2}{|c|}{ Температура T, K } & 320 & 205 & 143 \\
\hline \multicolumn{2}{|c|}{$\begin{array}{l}\text { Пространственная } \\
\text { группа }\end{array}$} & Pnma & Pnma: - 1ss & $\operatorname{Pn} 2{ }_{1} \mathrm{a}$ \\
\hline \multirow{3}{*}{$\begin{array}{l}\text { Параметры } \\
\text { ячейки }\end{array}$} & $a, \AA$ & $9.2759(3)$ & $27.681(2)$ & $27.603(3)$ \\
\hline & $b, \AA$ & $7.2926(2)$ & $7.2557(5)$ & $7.2328(8)$ \\
\hline & $c, \AA$ & $12.7523(4)$ & $12.6613(7)$ & $12.616(1)$ \\
\hline \multicolumn{2}{|c|}{ Объем ячейки V $\AA^{3}$} & $862.64(5)$ & $2543.0(3)$ & $2518.7(5)$ \\
\hline \multicolumn{2}{|c|}{ Формульное число $Z$} & 4 & 12 & 12 \\
\hline \multicolumn{2}{|c|}{$\mathrm{V} / \mathrm{Z}, \AA^{3}$} & 215,66 & 211,92 & 209,92 \\
\hline
\end{tabular}

Полученные при различных температурах значения отношения V/Z позволяют сделать оценку коэффициента объемного расширения ( $\beta$ ) частиц $\mathrm{Rb}_{2} \mathrm{ZnCl}_{4}$, входящих в состав композита RS-46. Воспользовавшись данными, представленными в таблице, найдем коэффициент объемного расширения $\beta \approx 153 \cdot 10^{-6} \mathrm{~K}^{-1}$. Это очень близко к величине $\beta$ (рис. 2), определенной для монокристалла тетрахлорцинката рубидия [11]. 
В случае композитов RS-46 и RS-320 температурные зависимости $\beta$ лежат существенно ниже зависимости $\beta(\mathrm{T})$ для массивного $\mathrm{Rb}_{2} \mathrm{ZnCl}_{4}$ (рис. 2). Это обусловлено тем, что исследуемые композиты преимущественно состоят из щелочно-боросиликатного стекла, характеризующегося очень небольшим коэффициентом $\beta \leq 15 \cdot 10^{-6} \mathrm{~K}^{-1}$ в интервале температур $100-310$ K [12].

Принимая это во внимание, можно заключить, что внедренные в пористые матрицы частицы испытывают деформации растяжения при охлаждении.

Какие-либо особенности на зависимостях $\beta(\mathrm{T})$ для композитов RS-46 и RS-320 в окрестностях, как $\mathrm{T}_{\mathrm{i}}$, так и $\mathrm{T}_{\mathrm{C}}$ не наблюдаются. Они едва заметны и для монокристалла $\mathrm{Rb}_{2} \mathrm{ZnCl}_{4}[11]$, что проиллюстрировано на рис.2.

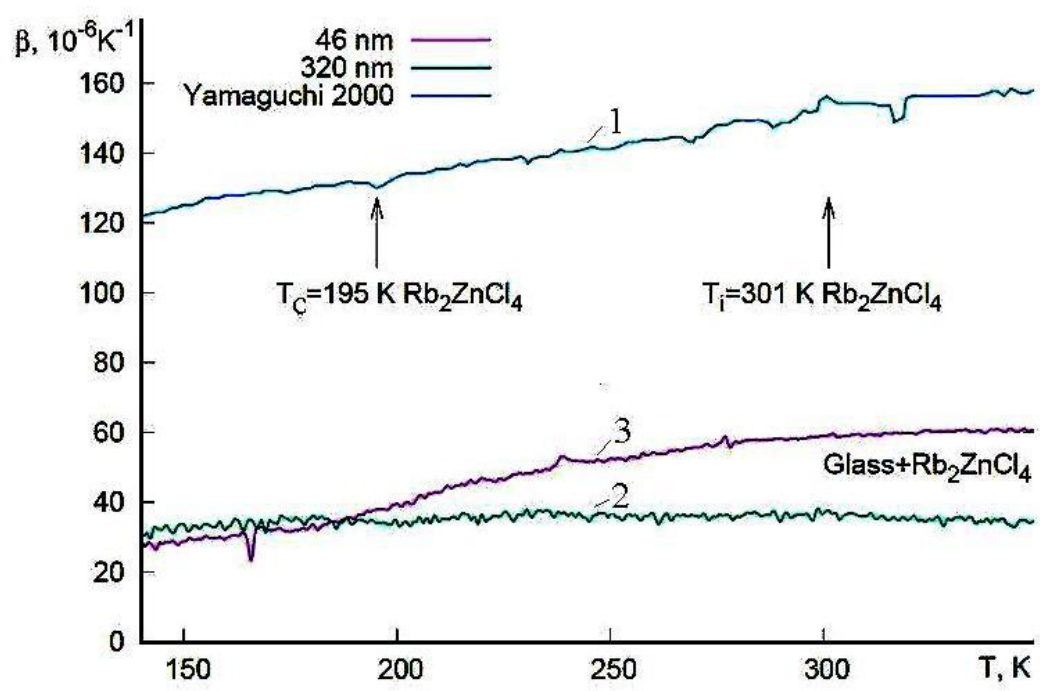

Рис. 2. Температурные зависимости коэффициента объемного теплового расширения для монокристалла $\mathrm{Rb}_{2} \mathrm{ZnCl}_{4}$ (1) [11] и композитов RS-320 (2) и $\mathrm{RS}-46$ (3).

Температурные зависимости диэлектрической проницаемости, полученные для образцов исследуемых материалов, представлены на рис. За. Зависимость $\varepsilon(T)$, полученная для массивного $\mathrm{Rb}_{2} \mathrm{ZnCl}_{4}$ имеет, по меньшей мере, два максимума. Первый - в окрестностях температуры $\mathrm{T}_{\mathrm{i}} \approx 307 \mathrm{~K}$ соответствует переходу из параэлектрической фазы в несоразмерную; второй - представляет собой отчетливый, несимметричный пик при температуре $\mathrm{T}_{\mathrm{C}} \approx 192 \mathrm{~K}$, соответствующий переходу между сегнетоэлектрической и несоразмерной фазами. В некотором интервале температур, лежащем ниже $\mathrm{T}_{\mathrm{C}}$, диэлектрическая проницаемость остается сравнительно высокой, образуя, так называемую область «плато», обусловленную высокой подвижностью доменных границ [13].

В монокристаллических образцах $\mathrm{Rb}_{2} \mathrm{ZnCl}_{4}$ «температура замораживания» аномально высокой подвижности доменов происходит при температуре $\mathrm{T}^{*} \approx 154$ К, которой 
соответствует слабая аномалия теплоемкости $[13,14]$ и максимум тангенса угла диэлектрических потерь $[13,15]$.

Температурные зависимости $\varepsilon$ для композитов RS-46 и RS-320 (рис. 3a) имеют почти одинаковый вид. Очевидно, это связано с тем, что несмотря на заметное различие среднего диаметра пор стеклянных матриц, доля внедренного вещества, а также средние размеры частиц $\mathrm{Rb}_{2} \mathrm{ZnCl}_{4}$ в порах различаются незначительно.

На зависимостях $\varepsilon(\mathrm{T})$ наблюдаются три аномалии около 160, 245 и 307 К. Максимум $\varepsilon$ около 307 К соответствует температуре, ниже которой появляется сверхструктурный рефлекс (2/3 2 0). Это обстоятельство позволяет связать данный максимум диэлектрической проницаемости с переходом между несоразмерной и параэлектрической фазами.

Для идентификации других особенностей диэлектрического отклика наряду с рентгенодифракционным экспериментом были проведены измерения теплоемкости образца композита RS-46 (рис. 3б). На зависимости $\mathrm{C}_{\mathrm{P}}(\mathrm{T})$, полученной в ходе нагрева, отчетливо видны два максимума. Размытый максимум, локализованный около 285 К, по-видимому связан с переходом из несоразмерной в параэлектрическую фазу. (Причины, вследствие которых его позиция не совпадает с позицией максимума $\varepsilon$ в окрестностях $\mathrm{T}_{\mathrm{i}} \approx 307 \mathrm{~K}$ требует дополнительных исследований, выходящих за рамки данной работы.)

Четкий пик СР вблизи 232 К имеет такой же вид, что и аномалия теплоемкости для монокристаллического тетрахлорцинката рубидия в окрестностях $\mathrm{T}_{\mathrm{C}}$ [14]. Это говорит о том, что данный максимум теплоемкости обусловлен сегнетоэлектрическим фазовым переходом. Вблизи его на зависимостях $\varepsilon(T)$, полученных в ходе охлаждения, композитов RS-46 и RS-320, регистрируется ступенькообразная аномалия. Однако ее температура лежит несколько выше позиции пика СР и составляет $\approx 245$ К.

Следует заметить, что на зависимостях $\varepsilon(\mathrm{T})$, полученных при нагреве образцов композиционных материалов отчетливых аномалий диэлектрического отклика, индицирующих сегнетоэлектрический фазовый переход не выявлено.

Обсудим размытый максимум диэлектрической проницаемости при $\mathrm{T}^{*}=160 \mathrm{~K}$. В отличие от аномалий $\varepsilon$ близи $\mathrm{T}_{\mathrm{i}}$ и $\mathrm{T}_{\mathrm{C}}$, максимум при $\mathrm{T}^{*}$ не сопровождается какой - либо особенностью на кривой $\mathrm{C}_{\mathrm{P}}(\mathrm{T})$. Это показывает, что он не связан со структурным фазовым переходом, а обусловлен некими процессами в доменной структуре. Такой максимум $\varepsilon$ около 160 К обнаружен для монокристалла $\mathrm{Rb}_{2} \mathrm{ZnCl}_{4}$ с высоким содержанием дефектов [14].

Существенным является то, что при измерениях в ходе циклического изменения температуры выше $\mathrm{T}_{0}$ имеет место заметный температурный гистерезис $\varepsilon$ (рис.3a). Похожий гистерезис характерен и для монокристаллического тетрахлорцинката рубидия, где он 
обусловлен метастабильными состояниями, связанными с пиннингом солитонов и доменных границ дефектами кристаллической решетки [13 - 15]. Ниже $\mathrm{T}_{0}$ гистерезисные явления не наблюдаются.

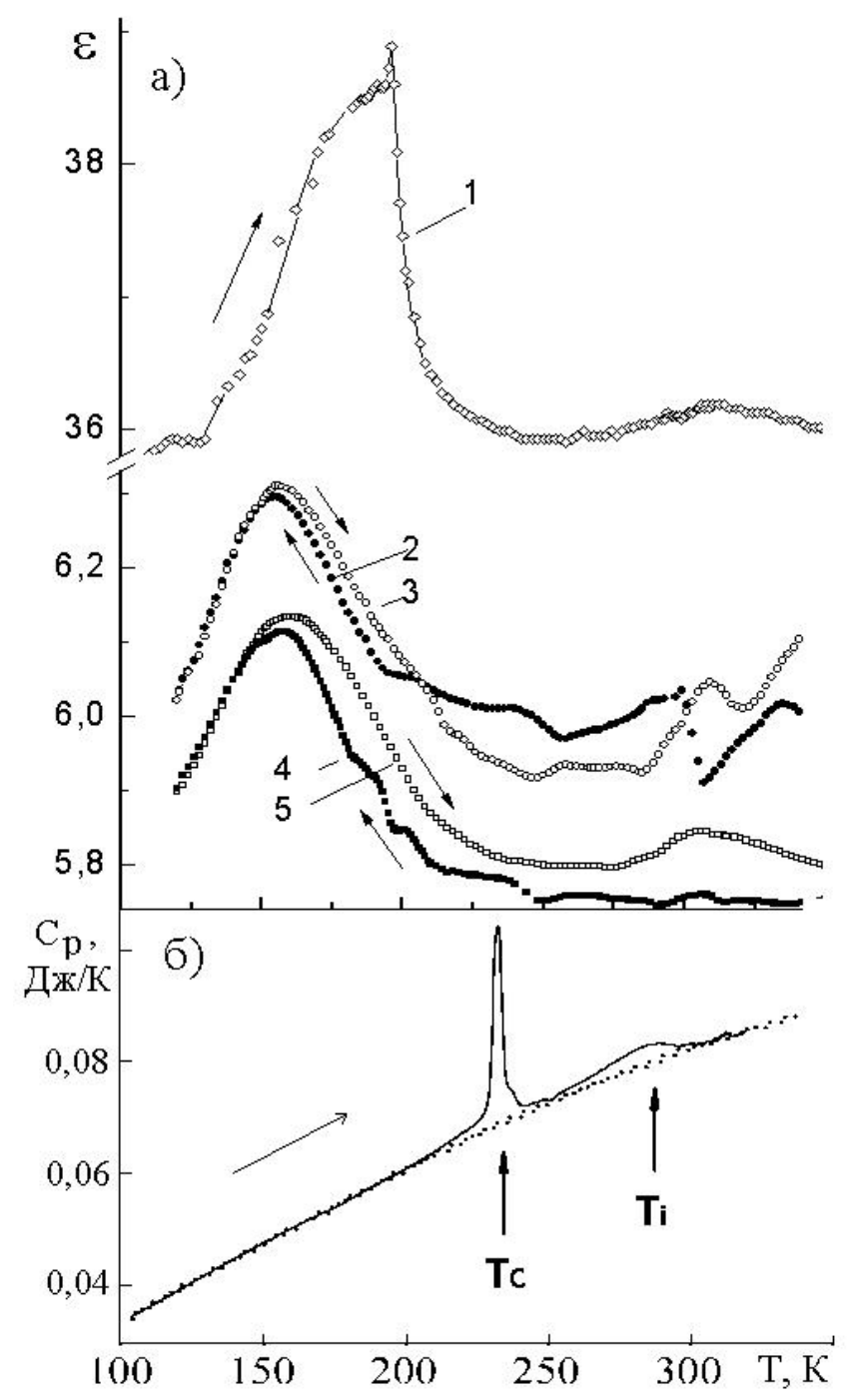

Рис. 3. (a) Температурные зависимости диэлектрической проницаемости для кристаллического образца $\mathrm{Rb}_{2} \mathrm{ZnCl}_{4}$ (1) и композитов RS-320 (2, 3) и RS-46 (4, 5), полученные в ходе нагрева $(1,3,5)$ и охлаждения $(2,4)$. (б) Температурная зависимость теплоемкости композита RS-46.

Подводя итог работе, основываясь на результатах проведенных исследований можно констатировать, что температура перехода в несоразмерную фазу $\mathrm{T}_{\mathrm{i}}$ в частицах $\mathrm{Rb}_{2} \mathrm{ZnCl}_{4}$, внедренных в пористые матрицы с нанометровым размером пор мало изменяется по сравнению с массивным монокристаллическим образцом. 
Анализ экспериментальных результатов показывает, что в сегнетоэлектрической фазе наночастиц $\mathrm{Rb}_{2} \mathrm{ZnCl}_{4}$ возникает подвижная доменная структура, которая «замораживается» при $\mathrm{T}_{0} \approx 155 \mathrm{~K}$, как и в объемном материале.

Наряду с этим температура сегнетоэлектрического фазового перехода в условиях ограниченной геометрии повышается на величину $\Delta \mathrm{T}_{\mathrm{C}} \approx 50$ К. Учитывая, что во внедренных частицах $\mathrm{Rb}_{2} \mathrm{ZnCl}_{4}$ при охлаждении появляются упругие напряжения растяжения $(\sigma)$, можно предположить, что именно они ответственны за повышение ТС. Оценим эти напряжения: $\sigma \approx \beta \mathrm{c} \Delta \mathrm{T} \approx 3,5 \cdot 10^{7}$ Па (здесь $\beta$ - коэффициента объемного расширения для монокристалла $\mathrm{Rb}_{2} \mathrm{ZnCl}_{4}$, c - среднее значение упругого модуля, полученное с использованием данных работы [16], и $\Delta \mathrm{T} \approx 100 \mathrm{~K}$ - разница между температурой внедрения вещества и $\mathrm{T}_{\mathrm{C}}$ ). Принимая во внимание, что $\mathrm{dT}_{\mathrm{C}} / \mathrm{dP}=-50 \mathrm{~K} / Г П а$ для $\mathrm{Rb}_{2} \mathrm{ZnCl}_{4}$ [17], получим $\Delta \mathrm{T}_{\mathrm{C}} \approx 1,5 \mathrm{~K}$, что существенно меньше наблюдаемого смещения температуры Кюри.

Для объяснения большого сдвига $\mathrm{T}_{\mathrm{C}}$ в частицах тетрахлорцинката рубидия, внедренных в пористые матрицы, учтем, что несоразмерная фаза характеризуется волной пространственных смещений атомов длинной $\lambda$ [18]. По мере удаления от $\mathrm{T}_{\mathrm{i}}$ в сторону низких температур $\lambda$ возрастает и при некоторой температуре она становится сопоставимой с размером частицы d. Тогда можно предположить, что сегнетоэлектрический фазовый переход в отдельной частице реализуется при условии $\lambda / 2 \approx \mathrm{d}$. Для оценки длины волны $\lambda$ воспользуемся экспериментальной температурной зависимостью параметра несоразмерности $\delta$, полученной в работе [8]. Для $\mathrm{T}=250 \mathrm{~K}$ параметр $\delta \approx 0,026$. Принимая, что период трансляции $\mathrm{C} \approx 1$ нм, находим $\lambda=\mathrm{C} / \delta \approx 38,5$ нм. Таким образом, действительно, вблизи температуры сегнетоэлектрического фазового перехода во внедренных в пористые матрицы частицах $\mathrm{Rb}_{2} \mathrm{ZnCl}_{4}$ выполняется соотношение $\lambda / 2 \approx \mathrm{d}$.

\section{СПИСОК ЛИТЕРАТУРЫ}

1. Rogazinskaya O. V., Milovidova S. D., Sidorkin A. S., Popravko N. G., Bosykh M. A., Enshina V. S. // Ferroelectrics, 2010. Vol. 397. P. 191.

2. Tarnavich V., Korotkov L., Karaeva O., Naberezhnov A., Rysiakiewicz-Pasek E. // Optica applicate, 2010. Vol. 40. P. 305.

3. Ciżman A., Rysiakiewicz -Pasek E., Kutrowska J., Poprawski R. // Solid State Phenomena, 2013. Vol. 200. P. 144.

4. E.A. Mikhaleva, I.N. Flerov, A. V. Kartashev, et al. // J. Mater Sci., 2018. V. 53. P.12132. 
5. E. A. Mikhaleva, I. N. Flerov, A. V. Kartashev, et al. //Ferroelectrics, 2017, V. 513. P. 44.

6. Fokin A.V., Kumzerov Yu. A., Okuneva N.M., Naberezhnov A. A., Vakhrushev S. B. // Phys. Rev. Lett., 2002. Vol. 89. P. 175503.

7. Бескровный А.И., Василовский С.Г., Вахрушев С.Б., Курдюков Д.А., Зворыкина О.И., Набережнов А.А., Окунева Н.М., Tovar M., Rysiakiewicz-Pasek E., Jagus P. // ФТТ, 2010. T. 52. C. 1021.

8. Багаутдинов Б.Ш., Шехтман В.Ш. // ФТТ, 1999. Т. 41. С. 1084.

9 Bruker AXS TOPAS V4, General profile and structure analysis software for powder diffraction data - User's Manual, Bruker AXS, Karlsruhe, Germany (2008).

10. A.V. Kartashev, I.N. Flerov, N.V. Volkov, K.A. Sablina. Phys. Solid State 50, 2115 (2008).

11. Yamaguchi T., Shimizu F. // Fermelectrics. 2000. V. 237. P. 201.

1 2. Е.А. Михалева, И.Н. Флёров, А.В. Карташев и др. //ФТТ, 2018. т. 60. Вып. 7. C.1328.

13. Гриднев С.А., Горбатенко В.В., Прасолов Б.Н. // Кристаллография, 1997. Т.42. №4. C. 730.

14. Струков Б.А., Белов А.А.,Горшков С.Н. и др. //Изв. АН СССР. Сер.физ., 1991. Т. 55. №3. C. 470.

15. Gridnev S.A., Shuvalov L.A., Gorbatenko V.V., et al. //Ferroelectrics. 1993. V. 140. P. 145.

16. Hirotsu S.H., Toyta K. Hamano K. // J. Phys. Soc. Jap. 1979. V. 46. P. 1389.

17. Gesi K. // Ferroelectrics. 1985. V. 64. P. 97.

18.

\section{X-Ray, Dielectric and Thermophysical Studies of Rubidium Tetrachlorozincate Within Porous Glasses.}

